\title{
Bem-Estar Materno pela Perspectiva do Design: Uma Revisão Sistemática da Produção Bibliográfica
}

\author{
Maternal Well-Being through the Design Perspective: A Systematic Review of \\ Bibliographic Production
}

COSTA, Filipe C. X.; Doutor; Programa de Pós Graduação em Design, UNISINOS

fcampelo@unisinos.br

SANT'ANNA, Paula A. G.; Mestranda; Programa de Pós Graduação em Design, UNISINOS

paula@inch.com.br

WOLFF, Fabiane; Doutora; Programa de Pós Graduação em Design, UNISINOS

fabiwolff@unisinos.br

\section{Resumo}

O design está presente na mediação entre as pessoas e o seu ambiente através de produtos e serviços com os quais interagem. Nesse cenário, o design para o bem-estar é uma área que propõe contribuir para a ampliação da percepção de felicidade dos indivíduos em seus específicos contextos. A presente revisão sistemática coletou e analisou estudos datados entre 2007 e 2017 na base de dados EBSCO e que podem servir como referência para projetos de design para o bemestar para mulheres que vivenciam a maternidade. A partir deste trabalho, foi possível observar que, apesar da maternidade ser um período de vulnerabilidade emocional amplamente discutido na literatura, abordagens para o bem-estar das mães a partir do design ainda são escassas.

Palavras Chave: positive design; bem-estar; maternidade.

\begin{abstract}
Design mediates people and their environment through the products and services with which they interact. In this scenario, design for well-being is an area that proposes to contribute to the expansion of individuals' perception of happiness in their specific contexts. The present systematic review collected and analyzed studies dating from 2007 to 2017 in the EBSCO database that can be used as a reference for design for well-being projects for women experiencing motherhood. Based on this research, it was possible to observe that, although maternity is a period of emotional vulnerability widely discussed in the literature, design approaches to mothers are still scarce.
\end{abstract}

Keywords: positive design; well-being; motherhood. 


\section{Introdução}

O design é cada vez mais explorado não apenas pelo seu potencial de beneficiar empresas e consumidores, mas também pelas mudanças positivas que pode causar aos indivíduos e à sociedade como um todo (MANZINI, 2015; DESMET E POHLMEYER, 2013; TROMP, HEKKERT E VERBEEK, 2011). Nessa perspectiva, o termo design para o bem-estar busca maneiras de contribuir para o bem-estar subjetivo das pessoas através de projetos que tenham como ponto de partida os benefícios que eles trarão aos indivíduos a longo prazo. Assim, é possível definir perfis de pessoas que poderiam ser favorecidas por projetos dessa finalidade.

Esta revisão sistemática partiu do questionamento: "qual a produção científica sobre o bem-estar materno pela perspectiva do design entre 2007 e 2017?". Para tanto, delimitou-se como público as mulheres que vivenciam a maternidade. O objetivo do trabalho é buscar e analisar estudos científicos que abordem a maternidade pela perspectiva do design para o bemestar. A intenção é compreender de que maneira os autores que exploram esse novo campo estão abordando o design como potencial ferramenta de amplificação ao bem-estar das mulheres durante a maternidade, período de alta vulnerabilidade emocional. Além disso, suas derivações e implicações podem servir como parâmetro para futuros estudos de design orientados para outros contextos sociais.

Justifica-se essa revisão por três motivos: (i) pela relevância do tópico da maternidade, que é vastamente abordado na literatura mundial por áreas como Medicina e Psicologia, sem, entretanto, apresentar consistência nos estudos do campo do design, (ii) por ser a maternidade um tema produtivo para a área do design para o bem-estar, já que esse período ocasiona à mulher fortes transformações emocionais, abrindo possibilidades para contribuições do design para esse público e (iii) por tratar-se do tema pesquisado pela autora do presente estudo em sua dissertação de mestrado, de forma que esta revisão sistemática será anexada à mesma.

A metodologia utilizada foi a revisão sistemática, por sua capacidade de compilação objetiva e baseada em evidências (KHAN ET AL., 2003), frente à vasta quantidade de publicações e dados disponíveis para coleta atualmente. Como fonte de pesquisa bibliográfica, optou-se pela utilização da plataforma EBSCO.

\section{Revisão Teórica}

\subsection{Design para o Bem-Estar}

Diferentes autores têm se dedicado a investigar como o design pode contribuir na mediação da experiência entre produtos e serviços com o usuário no intuito de estimular o seu bem-estar. Algumas abordagens assumem o termo de forma ampla, incluindo as diretrizes do social design (STEEN, 2016; TROMP, 2013; MANZINI, 2007), que propõe que a melhora da vida dos indivíduos se dá a partir dos impactos positivos gerados à sociedade. Essa visão aborda o design como uma ferramenta capaz de solucionar problemas da atualidade, aliando objetivos sociais e comerciais nos projetos (COTTAM E LEADBEATER, 2004), como o redesenho de novos sistemas de saúde.

O positive design é outra vertente que também aborda o design para o bem-estar, entretanto, seu foco é na contribuição à felicidade - ou bem-estar subjetivo - dos indivíduos (DESMET E POHLMEYER, 2013). Sua estrutura abrange três componentes: prazer, significado pessoal e virtude. Cada componente representa um ingrediente para estimular a felicidade, os 
quais devem ser contemplados pelo projeto de design para ajudar o indivíduo a desempenhar o seu melhor potencial. O maior interesse do positive design não é compreender os produtos, e sim o uso que as pessoas fazem deles, pois é nessa interatividade que torna-se possível estimular e engajar atividades significativas.

O primeiro componente do positive design é o design para o prazer, que ocorre em momentos de afeto positivo e ausência de afeto negativo, e refere-se aos prazeres instantâneos que uma experiência promove ao usuário. Já o design para significado pessoal decorre da felicidade que conecta a experiência às aspirações e objetivos da pessoa a longo prazo, ao invés dos instantâneos. Por fim, o design para virtude influencia o caráter do indivíduo, fazendo-o sentir-se uma pessoa moralmente boa (DESMET E POHLMEYER, 2013).

A partir dos elementos citados acima, o positive design sugere cinco princípios a serem utilizados como diretrizes projetuais: 1) design orientado às possibilidades: propõe o estímulo à excelência do design, ao invés da redução de suas deficiências; 2 ) equilíbrio: sugere o equilíbrio entre os três elementos do positive design, não no sentido de explorá-los de forma equivalente, e sim evitando incongruência entre eles; 3) ajuste pessoal: cada indivíduo possui interesses, preferências e motivações próprias, portanto o design deve ser centrado no perfil desse usuário, propondo estímulos a partir dessas características; 4) envolvimento ativo do usuário: a pessoa desempenha um papel importante na evolução do seu bem-estar, sendo assim, o design deve estimular seu senso de esforço e contribuição na experiência; 5) impacto a longo prazo: a partir do engajamento do usuário em experiências que o conectam com seus objetivos a longo prazo, é possível ampliar seu bem-estar-subjetivo.

De uma maneira geral, a principal diferença das teorias do design para o bem-estar às do design "tradicional" é que elas consideram que cada indivíduo possui características únicas motivações, preferências, habilidades e necessidades próprias (STEEN, 2016). Assim, o foco do design desloca-se da preocupação entre forma e função para um nível de contribuição a longo prazo ao indivíduo, considerando suas percepções de prazer, virtude e significado pessoal e, assim, apoiando sua evolução e potencial individuais.

Uma vez que o foco do design para o bem-estar é propor soluções que causem impacto positivo ao indivíduo a longo prazo, é possível buscar oportunidades de projeto focadas em grupos de pessoas de acordo com seus específicos contextos. No presente trabalho, as mães - mulheres que vivenciam a maternidade - foram definidas como o público a ser observado pela perspectiva do design para o bem-estar. Essa escolha foi pautada pelas motivações da autora ao compreender, a partir de sua pesquisa, que o contexto da maternidade é um palco de exploração frutífero à área do design para o bem-estar, já que confere às mulheres inúmeras transformações psicológicas.

\subsection{Contexto da Maternidade}

Apesar de ser geralmente percebida com muita alegria e emoções positivas, a maternidade é complexa e multifacetada, e pode representar desafios, ansiedade e ambiguidade para a mulher frente às situações de incerteza, stress e insegurança com que se depara (CARRIGAN E SZMIGIN, 2004). Além disso, a maternidade carrega consigo fortes significados culturais e ideologias sociais (HAGER, 2011), fato que motiva alguns autores a atribuírem parte da carga emocional advinda deste período à idealização da maternidade como uma construção social (HAGER, 2011; AZEVEDO E ARRAIS, 2006; GREINERT E MILANI, 2015). 
O primeiro ano da maternidade representa uma fase de transição universal à mulher (CURRIE, 2009). As transformações vivenciadas nesse período ocorrem em diversas esferas de sua vida. Durante a gravidez em si, sucedida pelo parto, iniciam as mudanças físicas que configuram uma nova relação da mulher com o seu corpo. Em paralelo, oscilações hormonais advindas da amamentação favorecem novas sensações, como instabilidades de humor. Com o nascimento de um bebê, surge também uma nova organização psíquica na vida da mulher, denominada constelação da maternidade, a qual provocará nela uma série de sentimentos, desejos e ações, constituindo-se no eixo organizador do psiquismo materno (STERN E VERONESE, 1997).

A maternidade se estabelece, essencialmente, a partir de um contexto muito íntimo, que é o da dinâmica entre mãe e filho, de acordo com ambas características individuais e experiências vivenciadas, paralelamente influenciado por sua conjuntura sociocultural. As bruscas mudanças no estilo de vida da mulher - que demarcam o antes e o depois da maternidade - envolvem imprevisibilidades, descobrimentos, adaptações e inseguranças, estabelecendo os hábitos de uma nova relação que envolve o reconhecimento da díade mãe e filho, sua jornada de cuidados, o confronto entre as expectativas e a realidade, as tarefas domésticas, a abstinência de sono, a nova configuração profissional, conjugal e social da mulher, entre tantos outros (PARKER, 1997).

Nesse complexo cenário da maternidade, é provável que a sensação mais forte que se apresente seja a ambivalência (PARKER, 1997). Frente às necessidades de decisão diárias que envolvem a vida da mãe e do filho, as múltiplas opções disponíveis que se opõem mutuamente podem causar à mulher uma carga emocional conflituosa. Além disso, as pressões culturais sob as quais é exercida a maternidade podem ser associadas ao sentimento de incapacidade de adequação aos critérios estabelecidos, causando ansiedade, culpa e amplificação dos conflitos emocionais (MANENTE, 2014).

Segundo Parker (1997), o próprio reconhecimento da abrangência da ambivalência materna por parte das mulheres já poderia ajudá-las a amenizar os prejuízos causados pela mesma em seu bem-estar. Entretanto, as abordagens na literatura a esse respeito são escassas, e discutem, em sua maioria, o bem-estar da criança, muitas vezes o atribuindo ao desempenho materno (PARKER, 1997).

É de acordo na literatura o quão fundamental é observar os riscos que o estado emocional prejudicado de uma mulher pode causar ao bebê, especialmente se desenvolvida a depressão pósparto. A patologia, que atinge uma em quatro mulheres no Brasil (FILHA ET AL., 2016), é a mais comum dentre os transtornos mentais do período e pode causar ao bebê transtornos cognitivos severos. Entretanto, para que patologias como essa sejam prevenidas, é necessário questionar como é possível contribuir para o bem-estar materno, ocasionando, assim, um melhor desempenho da experiência da maternidade.

Para tanto, é necessário compreender o cenário emocional em que a maternidade se estabelece, ou seja, quem é a mulher que se torna mãe, quais suas percepções, preferências e motivações próprias. A proposta do design para o bem-estar, conforme apresentado anteriormente, é justamente a de criar estratégias que contribuam para a felicidade de um indivíduo através de soluções que explorem maneiras de amplificar o seu bem-estar subjetivo (DESMET E POHLMEYER, 2013). 


\section{Metodologia}

O presente trabalho se estruturou a partir de uma revisão sistemática que coletou, selecionou e examinou todas as publicações que se enquadram nos critérios elegíveis do questionamento: "qual a produção científica sobre o bem-estar materno pela perspectiva do design entre 2007 e 2017 na base de dados EBSCO?". Procurou-se, assim, verificar a existência de publicações relevantes ao tema e compreender suas abordagens e contribuições, bem como futuras oportunidades para a área.

Frente à vasta quantidade de publicações e dados disponíveis para coleta na internet atualmente, a revisão sistemática é um método útil para pesquisa e seleção de dados baseados em evidências (KHAN ET AL., 2003). De acordo com os autores, é possível realizar uma revisão sistemática a partir de cinco passos: 1) formulação da questão da revisão de forma clara e objetiva; 2) identificação dos trabalhos relevantes a partir de uma busca extensa e abrangente; 3 ) avaliação da qualidade dos estudos conforme sua elegibilidade à questão levantada; 4) resumo das evidências, comparando suas diferenças e conclusões em comum; 5) interpretação dos resultados de acordo com os objetivos da pesquisa.

A plataforma EBSCO foi escolhida para a realização do levantamento bibliográfico pela abrangência e atualização contínua de suas bases de dados. Sendo a maternidade um assunto de vasta presença na literatura, julgou-se interessante investigar o conteúdo disponibilizado por uma plataforma que reúne publicações das mais variadas áreas de conhecimento.

O período da investigação delimitou-se nos últimos 10 anos, considerando a intenção de encontrar publicações que configurem o estado da arte da área, e também pelo fato de que o design para o bem-estar é ainda um campo relativamente novo (STEEN, 2016). Quanto ao tipo dos documentos, foram considerados os periódicos científicos e revistas acadêmicas. Todas as buscas foram realizadas a partir do idioma inglês e os resultados foram salvos em formato digital.

Quanto às palavras procuradas, foram sendo definidas diferentes combinações, das mais específicas às mais abrangentes, à medida em que não se alcançaram resultados relevantes nas buscas iniciais. Optou-se por procurar os termos como título, em razão do termo design ter inúmeros significados na língua inglesa, o que direciona uma pesquisa de palavras-chave, resumo ou texto completo, em grande parte, para diferentes contextos de utilização da palavra, como: desenho, esboço, modelo, planejamento, planta, projetos em geral, etc. Algumas palavras, como well-being e wellness, assim como mother e new mothers, foram utilizadas de forma conjunta na busca, por serem sinônimos em alguns contextos.

Assim, a análise sistemática foi estruturada conforme os critérios abaixo:

a. Base de dados: EBSCO

b. Critério de escolha: a plataforma foi escolhida por sua dimensão e abrangência na literatura científica

c. Critério de busca: Foram considerados artigos publicados em periódicos científicos e revistas acadêmicas com os títulos: 1 ) design e well-being ou wellness e mother ou new mothers, o qual resultou em zero publicação; 2) design e mother ou new mothers, o qual resultou em 25 publicações; 3) well-being ou wellness e new mothers, o qual resultou em cinco publicações. 
d. Período: 2007 a 2017

\section{Resultados}

A partir da estrutura metodológica da revisão sistemática, a busca foi realizada em três etapas na plataforma EBSCO. A Tabela 1 resume as três etapas da pesquisa a partir das palavras utilizadas e o total de artigos publicados, relevantes ou não ao tema deste trabalho.

Tabela 1 - Pesquisas de artigos publicados na plataforma EBSCO

\begin{tabular}{|c|c|c|c|}
\hline & $\begin{array}{c}\text { Palavras pesquisadas no } \\
\text { título }\end{array}$ & Total de artigos publicados & $\begin{array}{l}\text { Total de artigos publicados } \\
\text { relevantes ao tema }\end{array}$ \\
\hline Pesquisa 1 & $\begin{array}{c}\text { design } \\
\text { well-being ou wellness } \\
\text { mother ou new mothers }\end{array}$ & 0 & 0 \\
\hline Pesquisa 2 & $\begin{array}{c}\text { design } \\
\text { mother ou new mothers }\end{array}$ & 25 & 0 \\
\hline Pesquisa 3 & $\begin{array}{c}\text { well-being ou wellness } \\
\text { new mothers }\end{array}$ & 5 & 3 \\
\hline
\end{tabular}

Fonte: Elaborado pelos autores, com base na pesquisa realizada

A primeira pesquisa foi realizada com a intenção de encontrar publicações que tratassem diretamente do assunto desejado: o bem-estar materno pela perspectiva do design. Para fins de maior alcance, optou-se por não limitar o uso do termo design para o bem-estar de forma conjunta, já que as palavras design e well-being utilizadas individualmente poderiam gerar composições relevantes. Contudo, conforme mostra a Tabela 1, não houve resultado. As pesquisas 2 e 3, por outro lado, resultaram nas publicações que constam nos Quadros 1 e 2, com os respectivos ano de publicação, periódico e título.

Quadro 1 - Resultados da Pesquisa 2

\begin{tabular}{l|l|l}
\hline 2008 & Behavior Genetics & $\begin{array}{l}\text { The cross-generational mother-daughter-aunt-niece design: establishing } \\
\text { validity of the MDAN design with NLSY fertility variables }\end{array}$ \\
\hline 2008 & Journal of Women's Health & $\begin{array}{l}\text { Active Mothers Postpartum (AMP): Rationale, Design, and Baseline } \\
\text { Characteristics }\end{array}$ \\
\hline 2010 & $\begin{array}{l}\text { Journal of Nutrition } \\
\text { Education \& Behavior }\end{array}$ & $\begin{array}{l}\text { Design and Outcomes of a Mothers In Motion Behavioral Intervention Pilot } \\
\text { Study }\end{array}$ \\
\hline 2010 & $\begin{array}{l}\text { International Journal of } \\
\text { Production Research }\end{array}$ & $\begin{array}{l}\text { Two-staged guillotine cut, two-dimensional bin packing optimisation with } \\
\text { flexible bin size for steel mother plate design }\end{array}$ \\
\hline 2011 & $\begin{array}{l}\text { BMC Medical Research } \\
\text { Contempology }\end{array}$ & $\begin{array}{l}\text { Building capacity for evidence generation,synthesis and implementation to } \\
\text { improve the careof mothers and babies in South East Asia: methodsand } \\
\text { design of the SEA-ORCHID Project using alogical framework approach }\end{array}$ \\
\hline 2011 & The Kesho Bora Study Group & $\begin{array}{l}\text { Safety and effectiveness of antiretroviral drugs during pregnancy, delivery } \\
\text { and breastfeeding for prevention of mother-to-child transmission of HIV-1: } \\
\text { the Kesho Bora Multicentre Collaborative Study rationale, design, and } \\
\text { implementation challenges }\end{array}$ \\
\hline
\end{tabular}




\begin{tabular}{|c|c|c|}
\hline & & $\begin{array}{l}\text { and breastfeeding for prevention of mother-to-child transmission of HIV-1: } \\
\text { The Kesho Bora Multicentre Collaborative Study rationale, design, and } \\
\text { implementation challenges }\end{array}$ \\
\hline 2012 & $\begin{array}{l}\text { American Journal of } \\
\text { Epidemiology }\end{array}$ & $\begin{array}{l}\text { A Trigger-based Design for Evaluating the Safety of In Utero Antiretroviral } \\
\text { Exposure in Uninfected Children of Human Immunodeficiency Virus-Infected } \\
\text { Mothers }\end{array}$ \\
\hline 2012 & $\begin{array}{l}\text { Maternal \& Child Health } \\
\text { Journal }\end{array}$ & $\begin{array}{l}\text { Using Qualitative Methods to Design a Culturally Appropriate Child Feeding } \\
\text { Questionnaire for Low-Income, Latina Mothers }\end{array}$ \\
\hline 2012 & Prevention Science & $\begin{array}{l}\text { The Family Spirit Trial for American Indian Teen Mothers and Their Children: } \\
\text { CBPR Rationale, Design, Methods and Baseline Characteristics }\end{array}$ \\
\hline 2013 & Journal Of Cancer Education & $\begin{array}{l}\text { From adolescent daughter to mother: exploring message design strategies } \\
\text { for breast and cervical cancer prevention and screening }\end{array}$ \\
\hline 2013 & $\begin{array}{l}\text { Razi Journal of Medical } \\
\text { Sciences }\end{array}$ & $\begin{array}{l}\text { Designing and evaluating educational intervention based on health belief } \\
\text { model on promoting the preventive behaviors of children with urinary tract } \\
\text { infections in mothers under the age of } 6 \text { years old. }\end{array}$ \\
\hline 2014 & BMC Public Health & $\begin{array}{l}\text { A community based prevention of weight gain intervention (Mothers In } \\
\text { Motion) among young low-income overweight and obese mothers: design } \\
\text { and rationale }\end{array}$ \\
\hline 2014 & $\begin{array}{l}\text { Journal of Community } \\
\text { Health }\end{array}$ & $\begin{array}{l}\text { The Peru Cervical Cancer Screening Study (PERCAPS): The Design and } \\
\text { Implementation of a Mother/Daughter Screen, Treat, and Vaccinate Program } \\
\text { in the Peruvian Jungle }\end{array}$ \\
\hline 2014 & $\begin{array}{l}\text { Indian Journal Of } \\
\text { Psychological Medicine }\end{array}$ & $\begin{array}{l}\text { Enhancing Mother Infant Interactions through Video Feedback Enabled } \\
\text { Interventions in Women with Schizophrenia: A Single Subject Research } \\
\text { Design Study }\end{array}$ \\
\hline 2014 & $\begin{array}{l}\text { Journal Of Acquired Immune } \\
\text { Deficiency Syndromes }\end{array}$ & $\begin{array}{l}\text { Cluster randomized trial on the effect of mother support groups on } \\
\text { retention-in-care and PMTCT outcomes in Zimbabwe: study design, } \\
\text { challenges, and national relevance }\end{array}$ \\
\hline 2014 & $\begin{array}{l}\text { Value In Health: The Journal } \\
\text { Of The International Society } \\
\text { For Pharmacoeconomics } \\
\text { And Outcomes Research }\end{array}$ & $\begin{array}{l}\text { Predictive Modelling for Optimal Target Population and Real-World Study } \\
\text { Design: An Example In Mother-To-Child Transmission of HIV }\end{array}$ \\
\hline 2014 & BioMed Centrals & $\begin{array}{l}\text { Motivational interviewing with American Indian mothers to prevent early } \\
\text { childhood caries: study design and methodology of a randomized control trial }\end{array}$ \\
\hline 2015 & $\begin{array}{l}\text { Journal of Child \& Family } \\
\text { Studies }\end{array}$ & $\begin{array}{l}\text { Giving Voice to Working Mothers: A Consumer Informed Study to Program } \\
\text { Design for Working Mothers }\end{array}$ \\
\hline 2015 & BMC Pregnancy \& Childbirth & $\begin{array}{l}\text { Opportunities to improve postpartum care for mothers and infants: design of } \\
\text { context-specific packages of postpartum interventions in rural districts in } \\
\text { four sub-Saharan African countries }\end{array}$ \\
\hline 2015 & Contemporary Clinical Trials & $\begin{array}{l}\text { Rationale and design of a comparative effectiveness trial to prevent type } 2 \\
\text { diabetes in mothers and children: The ENCOURAGE healthy families study }\end{array}$ \\
\hline 2016 & $\begin{array}{l}\text { Journal Of Acquired Immune } \\
\text { Deficiency Syndromes }\end{array}$ & $\begin{array}{l}\text { Mother Infant Retention for Health (MIR4Health): Study Design, } \\
\text { Adaptations, and Challenges With PMTCT Implementation Science Research }\end{array}$ \\
\hline 2016 & $\begin{array}{l}\text { Annals of King Edward } \\
\text { Medical University }\end{array}$ & $\begin{array}{l}\text { Weaning Practices and Perceptions of Mothers Residing in Urban Slums of } \\
\text { Lahore, Pakistan: A Focus Group Design }\end{array}$ \\
\hline 2016 & $\begin{array}{l}\text { JAIDS: Journal of Acquired } \\
\text { Immune } \\
\text { Syndromes }\end{array}$ & $\begin{array}{l}\text { Mother Infant Retention for Health (MIR4Health): Study Design, Adaptations, } \\
\text { and Challenges With PMTCT Implementation Science Research }\end{array}$ \\
\hline 2017 & Contemporary Clinical Trials & $\begin{array}{l}\text { Evaluation of mHealth strategies to optimize adherence and efficacy of } \\
\text { Option B+ prevention of mother-to-child HIV transmission: Rationale, design } \\
\text { and methods of a 3-armed randomized controlled trial }\end{array}$ \\
\hline
\end{tabular}

Fonte: Elaborado pelos autores, com base na pesquisa realizada 
Conforme mostra o Quadro 2, a segunda pesquisa foi realizada de forma mais abrangente, sem as palavras well-being e wellness. O objetivo foi investigar a existência de estudos que pudessem tratar essencialmente de abordagens de design em relação às mães, o que, ainda assim, poderia fundamentar futuras pesquisas de design para o bem-estar para esse público. Nesse cenário, entretanto, ficou evidente o predomínio de publicações que abordam a maternidade e o bem-estar pelo viés da medicina. Das 25 publicações encontradas, oito têm como foco a prevenção ao vírus HIV, quatro discutem questões como nutrição e sobrepeso, enquanto as demais abordam assuntos como câncer, diabetes, fertilidade, desmame, entre outros. Fica perceptível, assim, que o uso do termo design nesses casos diz respeito ao design enquanto modelo, desenho ou solução para um problema, e não como um instrumento projetual.

Quadro 2 - Resultados da Pesquisa 3

\begin{tabular}{l|l|l}
\hline Ano & \multicolumn{1}{|c|}{ Periódico } & \multicolumn{1}{c}{ Título da Publicação } \\
\hline 2008 & $\begin{array}{l}\text { Birth: Issues in Perinatal } \\
\text { Care }\end{array}$ & $\begin{array}{l}\text { Postpartum physical symptoms in new mothers: their relationship to } \\
\text { functional limitations and emotional well-being }\end{array}$ \\
\hline 2009 & $\begin{array}{l}\text { Health Care for Women } \\
\text { International }\end{array}$ & $\begin{array}{l}\text { Managing motherhood: strategies used by new mothers to maintain } \\
\text { perceptions of wellness }\end{array}$ \\
\hline 2010 & Physical Therapy & $\begin{array}{l}\text { An exercise and education program improves well-being of new mothers: a } \\
\text { randomized controlled trial }\end{array}$ \\
\hline 2012 & $\begin{array}{l}\text { Maternal \& Child } \\
\text { Health Journal }\end{array}$ & $\begin{array}{l}\text { New Mothers and Media Use: Associations Between Blogging, Social } \\
\text { Networking, and Maternal Well-Being }\end{array}$ \\
\hline
\end{tabular}

Fonte: Elaborado pelos autores, com base na pesquisa realizada

Para a terceira pesquisa, optou-se por utilizar apenas o termo new-mothers junto a wellbeing e wellness. O motivo dessa escolha foi a ascensão do termo new mothers na literatura enquanto um grupo de mães primíparas (mães de um primeiro filho) que são observadas pelas características que as diferem das mães de mais de um filho, como pela sua maior suscetibilidade emocional decorrente da primeira experiência da maternidade. Nessa última investigação, cinco artigos emergiram, sendo que três publicações foram elegíveis aos critérios de seleção para o presente trabalho, pois seu escopo têm como objetivo central promover o bem-estar da mulher em contextos da maternidade. Apesar de não terem sido fundamentados no campo do design para o bem-estar, esses estudos buscam compreender ferramentas e estratégias que podem contribuir para a felicidade da mulher nesse período, e, portanto, possuem validade para este trabalho e serão analisados. $O$ quadro 3 compila as principais características das publicações:

Quadro 3 - Resultados dos artigos relevantes ao tema

\begin{tabular}{l|l|l}
\hline \multicolumn{1}{c|}{ Autor } & \multicolumn{2}{c}{ Estudo } \\
\hline \multirow{2}{*}{$\begin{array}{l}\text { Currie, Janet, } \\
2009\end{array}$} & Título & $\begin{array}{l}\text { Managing motherhood: strategies used by new mothers to maintain } \\
\text { perceptions of wellness. }\end{array}$ \\
\cline { 2 - 3 } & Objetivo & $\begin{array}{l}\text { Compreender as estratégias utilizadas por um grupo de mães em sua rotina } \\
\text { para manterem sua percepção de bem-estar. }\end{array}$ \\
\hline
\end{tabular}




\begin{tabular}{|c|c|c|}
\hline & Método & $\begin{array}{l}\text { Através de um estudo qualitativo exploratório fundamentado na Grounded } \\
\text { Theory, mães de crianças de até cinco anos foram convidadas a participarem } \\
\text { de entrevistas e grupos focais. }\end{array}$ \\
\hline & Resultado & $\begin{array}{l}\text { Foram criados três grandes categorias que reúnem estratégias em comum } \\
\text { relatadas pelas mães na busca da manutenção por seu bem-estar, o que } \\
\text { enfatiza a influência e papel das mulheres na ampliação do seu próprio } \\
\text { bem-estar. }\end{array}$ \\
\hline \multirow{4}{*}{$\begin{array}{l}\text { McDaniel, } \\
\text { Brandon; Coyne, } \\
\text { Sarah; } \\
\text { Holmes, Erin, } \\
2012\end{array}$} & Título & $\begin{array}{l}\text { New Mothers and Media Use: Associations Between Blogging, Social } \\
\text { Networking, and Maternal Well-Being. }\end{array}$ \\
\hline & Objetivo & $\begin{array}{l}\text { Examinar a associação existente entre a utilização de meios digitais por } \\
\text { parte das mães - blogs, redes sociais, websites, e mail, etc - e a sua } \\
\text { percepção de bem-estar. }\end{array}$ \\
\hline & Método & $\begin{array}{l}\text { Novas mães, com um filho de até dezoito meses, foram recrutadas para } \\
\text { participar de uma pesquisa online que foi mensurada através do software de } \\
\text { análise estrutural S.E.M. }\end{array}$ \\
\hline & Resultado & $\begin{array}{l}\text { A alta frequência de uso dos meios digitais ocasionou às mães o sentimento } \\
\text { de conexão estendida a seus amigos e familiares, e a consequente } \\
\text { percepção de suporte social ampliado, configurando-se influente na redução } \\
\text { do stress maternal. }\end{array}$ \\
\hline \multirow{4}{*}{$\begin{array}{l}\text { Hamilton, Kyra; } \\
\text { Kavanagh, David; } \\
\text { Connolly, Jennifer; } \\
\text { Davis, Leigh; } \\
\text { Fisher, Jane; } \\
\text { Halford, Kim; } \\
\text { Hides, Leanne; } \\
\text { Milgrom, } \\
\text { Jeannette; } \\
\text { Rowe, Heather; } \\
\text { Sanders, Davina; } \\
\text { Scuffham, Paul A; } \\
\text { Tjondronegoro, } \\
\text { Dian; } \\
\text { Walsh, Anne; } \\
\text { White, Katherine; } \\
\text { MWittkowski, Anja, } \\
\text { 2016 }\end{array}$} & Título & $\begin{array}{l}\text { Baby Steps - An Online Program Promoting the Well-Being of New Mothers } \\
\text { and Fathers: A Study Protocol. }\end{array}$ \\
\hline & Objetivo & $\begin{array}{l}\text { Comparar o impacto das duas versões do programa online Baby Steps na } \\
\text { redução da angústia, ampliação de bem-estar, satisfação na vida conjugal e } \\
\text { eficácia parental dos pais. }\end{array}$ \\
\hline & Método & $\begin{array}{l}\text { Casais primíparos foram selecionados para testar dois programas online, } \\
\text { sendo o primeiro focado apenas em informações sobre cuidado com o bebê, } \\
\text { enquanto o outro inclui conteúdo motivacional focado no bem-estar dos } \\
\text { pais. }\end{array}$ \\
\hline & Resultado & $\begin{array}{l}\text { O engajamento mútuo dos pais proposto pelo programa no desafio de lidar } \\
\text { e vencer juntos os desafios da parentalidade, através de uma plataforma } \\
\text { informativa, motivacional e persuasiva, parece ter sido percebida } \\
\text { positivamente pelos casais participantes do teste. }\end{array}$ \\
\hline
\end{tabular}

Fonte: Elaborado pelos autores, com base na pesquisa realizada

A partir do Quadro 3, é possível observar a natureza heterogênea dos estudos que foram selecionados. Apesar dos dois primeiros artigos não tratarem especificamente de projetos de design, eles podem servir como base teórica para estratégias que tenham como objetivo a amplificação do bem-estar materno, uma vez que abordam esse assunto pelos relatos das próprias mães.

$\mathrm{Na}$ primeira pesquisa, Currie (2009) investiga quais estratégias são utilizadas pelas mulheres que vivenciam a maternidade para gerenciarem seus estilos de vida e cultivarem sua percepção de bem-estar. Por meio de entrevistas e grupos focais com análise de dados e conclusão por saturação, seus achados foram agrupados em três principais categorias: 1) obtendo 
ajuda; 2) organizando a rotina; 3) planejando atividades pessoais fora. Por meio dessas estratégias, as mulheres entrevistadas procuram maneiras de auto gerenciarem suas rotinas e, consequentemente, desempenharem a maternidade de forma mais positiva. Para Currie (2009), as experiências descritas refletem princípios universais para a promoção do bem-estar e enfatizam o potencial de influência positiva da participação contínua do indivíduo no aumento do seu próprio bem-estar.

O estudo de McDaniel et al. (2012) também não apresenta um projeto específico a ser explorado na amplificação do bem-estar materno, entretanto, busca compreender de que maneiras os meios digitais podem influenciar de forma positiva aspectos emocionais da vida da mulher. Através de um questionário online, mães primíparas de bebês de até dezoito meses foram recrutadas para compartilhar as percepções que o uso de blogs, redes sociais, websites e emails causavam no seu desempenho materno e conjugal. O estudo revelou que, para muitas mães, a troca de informações compartilhadas pela internet com seus amigos e familiares representava uma sensação simbólica de suporte social, o que desempenhou papel positivo em suas vivências enquanto mãe e cônjuge. Para os autores, a associação entre o uso digital e o bem-estar na experiência da maternidade é um campo ainda pouco explorado, mas de vastas possibilidades na literatura científica.

Hamilton et al. (2016) apresentam o programa Baby Steps, formulado com o intuito de melhorar o desempenho dos pais e suas percepções de bem-estar. Os autores defendem que o bem-estar do pai e da mãe, quando prejudicados frente aos desafios e angústias do exercício da parentalidade, podem causar riscos a sua saúde mental, assim como à da criança e seu desenvolvimento. Entretanto, a maioria das pesquisas e serviços de apoio estão focados na mãe e no tratamento de patologias, deixando uma lacuna na oportunidade de exercitar o potencial de amplificação do bem-estar através do engajamento mútuo dos pais. Por meio de um programa online informativo, motivacional e persuasivo, o Baby Steps parece ser percebido como uma alternativa interessante de apoio emocional aos pais, utilizando sua relação como motivador e considerando as dinâmicas e estilos de vida atuais.

\section{Considerações Finais}

Considerando a abrangência de estudos a respeito da maternidade na literatura (CURRIE, 2009) e a relevância social do tema tanto para a mulher quanto para seu filho (HAGER, 2011; FILHA ET AL., 2016), em paralelo à emergente abordagem do design como instrumento transformador de contextos (MANZINI, 2014), é surpreendente que haja tão poucos estudos que relacionem o bem-estar materno ao design.

Entretanto, é válido considerar a contribuição que os artigos selecionados trouxeram ao tema aqui proposto. Para projetar para o bem-estar, é necessário compreender os diferentes contextos, percepções, preferências e motivações dos indivíduos (DESMET E POHLMEYER, 2013). Essa base teórica irá fundamentar as estratégias a serem tomadas para que seja possível criar uma conexão entre essas pessoas e as experiências que irão vivenciar através de projetos de design. Conhecer suas características únicas é o ponto de partida para prever cenários que irão engajá-las em situações significativas, as quais, a longo prazo, poderão estimular a percepção de amplificação do seu bem-estar.

Sendo assim, os três artigos analisados podem ser considerados úteis ao projetar para as mães pela perspectiva de seu bem-estar. Currie (2009) converte relatos em estratégias que podem ser utilizadas como ponto de partida para projetos de design. Por outro lado, McDaniel et al. 
(2012) investigam as significações da troca de experiência virtual na percepção de bem-estar da mãe, abrindo caminho para propostas que explorem experiências no meio virtual, que é o caso do terceiro estudo. Nele, Hamilton et al. (2016) experimentam conectar necessidades emocionais dos pais às possibilidades que as dinâmicas digitais oferecerem, por meio de uma plataforma projetada com a intenção de ampliar a percepção de bem-estar entre eles e seu filho.

Revisões sistemáticas futuras a respeito deste tema poderão ser realizadas por meio de outros bancos de dados e, até mesmo, por novas combinações de palavras, desde que a linha de pesquisa em design seja conduzida pelo olhar da maternidade. Novas descobertas serão válidas tanto aos estudos sobre a maternidade, quanto aos do design para o bem-estar.

A partir dos achados desta pesquisa, entende-se haver um campo de exploração disponível na conexão entre os temas de maternidade e design para o bem-estar, com potencial de contribuição para as respectivas áreas científicas. Com esse propósito, tal pesquisa está sendo conduzida pela autora do artigo no ambiente de seus grupos de pesquisa como forma de investigar, de maneira mais aprofundada e vinculada a processos projetuais, as contribuições do design ao bem-estar dos indivíduos, tendo como objeto de estudo, sequencialmente, a experiência da maternidade.

\section{Referências}

CARRIGAN, Marylyn; SZMIGIN, Isabelle. Time, uncertainty and the expectancy experience: An interpretive exploration of consumption and impending motherhood. Journal of Marketing Management, v. 20, n. 7-8, p. 771-798, 2004.

COTTAM, Hilary; LEADBEATER, Charles. RED paper 01: Health: Co-creating services. London: Design Council, 2004.

CURRIE, Janet. Managing motherhood: Strategies used by new mothers to maintain perceptions of wellness. Health Care for Women International, v. 30, n. 7, p. 653-668, 2009.

DESMET, Pieter; POHLMEYER, Anna E. Positive design: An introduction to design for subjective well-being. International Journal of Design, v. 7, n. 3, 2013.

FILHA, Mariza M. T. et al. Factors associated with postpartum depressive symptomatology in Brazil: the Birth in Brazil national research study, 2011/2012. Journal of affective disorders, v. 194, p. 159-167, 2016.

GREINERT, Bruna R. M.; MILANI, Rute G. (2015). Depressão pós-parto: uma compreensão psicossocial. Psicologia: teoria e prática, 17(1), 26-36.

HAGER, Tamar. Making sense of an untold story: A personal deconstruction of the myth of motherhood. Qualitative Inquiry, v. 17, n. 1, p. 35-44, 2011.

HAMILTON, Kyra et al. Baby Steps-an online program promoting the well-being of new mothers and fathers: A study protocol. JMIR research protocols, v. 5, n. 3, 2016.

KHAN, Khalid S. et al. Five steps to conducting a systematic review. Journal of the Royal Society of Medicine, v. 96, n. 3, p. 118-121, 2003.

MANENTE, Milena V. Maternidade para mães trabalhadoras: depressão pós-parto, stress, rede de apoio e conjugalidade. 2014. 125 f. Tese (mestrado) - Universidade Estadual Paulista Julio de Mesquita Filho, Curso de Pós-Graduação em Psicologia do Desenvolvimento e Aprendizagem. 
MANZINI, Ezio. Design research for sustainable social innovation. Design research now, p. 233245, 2007.

MANZINI, Ezio. Making things happen: Social innovation and design. Design Issues, v. 30, n. 1, p. 57-66, 2014.

MCDANIEL, Brandon T.; COYNE, Sarah M.; HOLMES, Erin K. New mothers and media use: Associations between blogging, social networking, and maternal well-being. Maternal and child health journal, v. 16, n. 7, p. 1509-1517, 2012.

PARKER, Rozsika; DE LIMA, Alice X.A mãe dividida: a experiência da ambivalência na maternidade. Rosa dos Tempos, 1997.

ROGAN, Frances et al. 'Becoming a mother' - developing a new theory of early motherhood. Journal of advanced nursing, v. 25, n. 5, p. 877-885, 1997.

STEEN, Marc. Organizing Design-for-Wellbeing Projects: Using the Capability Approach. Design Issues, v. 32, n. 4, p. 4-15, 2016.

STERN, Daniel N.; VERONESE, Maria Adriana V. A constelação da maternidade: o panorama da psicoterapia pais/bebê. Artes Médicas, 1997.

TROMP, Nynke; HEKKERT, Paul; VERBEEK, Peter-Paul. Design for socially responsible behavior: a classification of influence based on intended user experience. Design Issues, v. 27, n. 3, p. 3-19, 2011.

TROMP, Nynke; HEKKERT, Paul. Social implication design (SID): A design method to exploit the unique value of the artefact to counteract social problems. Umeå Institute of Design, 2014. 